\title{
Generalized Cotorsion Locally Compact Abelian Groups
}

\author{
N. I. KRYUCHKOV
}

ABstract - This paper is concerned with the generalization of the concept of cotorsion abelian group. A locally compact abelian group $L$ is called generalized cotorsion if $L$ contains a compact open subgroup $K$ such that the character group of $K$ and the group $L / K$ are cotorsion groups. Some properties and homological characteristics of generalized cotorsion groups are obtained. The classification problem of generalized cotorsion groups is discussed.

\section{Introduction}

Throughout, all groups will be abelian groups. This paper is concerned with the generalization of the well-known in abelian group theory concept of cotorsion group. The notion of cotorsion group was introduced and studied by Harrison [1]. Fuchs [2] and Nunke [3] discovered the same notion independently. A discrete group $A$ is called a cotorsion group if and only if $\operatorname{Ext}(Q, A)=0$, where $Q$ is the additive group of rational numbers. Cotorsion groups play an important role in the theory of Abelian groups. The category of compact abelian groups is dual to the category of discrete abelian groups by Pontrjagin duality theory. Therefore any concept in the theory of discrete abelian groups has a dual concept in the theory of compact groups and they are both specifications of some concepts in the theory of locally compact abelian groups. Every statement about discrete groups has its dual assertion in the theory of compact groups. Both of these are usually special cases some statement about locally compact abelian groups. In this paper we construct a new class of locally compact abelian groups, which contains the class of cotorsion groups and its dual. The objects of this new class are called generalized cotorsion groups. The class

(*) Indirizzo dell'A.: Department of Mathematics, Ryazan State University, Svobody, 46 Ryazan, 390048 Russia.

E-mail: kryuchkov.n@gmail.com 
of generalized cotorsion groups is enough large. It include the class of cotorsion groups and the class the duals of cotorsion groups. Every compact coreduced generalized cotorsion group is a character group some group of extensions. The class generalized cotorsion groups has a number interesting properties. Every extension of a compact coreduced group by a discrete reduced group may be connect with some generalized cotorsion group. Therefore, generalized cotorsion groups should play an important role in the theory of locally compact abelian groups.

In Section 2 the class of dual cotorsion groups is defined. A compact group $K$ is called dual cotorsion group if and only if the character group of the group $K$ is a discrete cotorsion group. Some properties of compact dual cotorsion groups are investigated.

In Section 3 we define the concept of generalized cotorsion groups. A locally compact group $L$ is called generalized cotorsion if $L$ contains an open dual cotorsion subgroup $K$, such that the factor group $L / K$ is a cotorsion group. We study properties of generalized cotorsion groups. The main results of this section are Theorem 3.3 and Theorem 3.7.

In Section 4 some homological properties of generalized cotorsion groups are investigated. The main statements of this section are Theorem 4.4 and Theorem 4.5 .

In Section 5 the problem of a classification of generalized cotorsion groups is discussed.

This paper relies heavily on the Pontrjagin Duality Theorem and related structural and character theoretic facts about locally compact groups. Material on discrete group theory, locally compact group theory, and Pontrjagin duality can be found in [4] - [7]. By $\mathcal{A}$ denote the class of discrete abelian groups and denote by $\mathcal{C}$ the class of compact abelian groups. By $\mathcal{L}$ denote the class of locally compact abelian groups.

By $\widehat{L}$ denote the character group of a locally compact group $L$ considering with its compact-open topology, so that it is a locally compact group. A compact group $K$ is called coreduced if the group $\widehat{K}$ is a reduced group. If $G$ and $H$ are locally compact groups and $f: G \rightarrow H$ is a continuous homomorphism we denote by $\widehat{f}: \widehat{H} \rightarrow \widehat{G}$ the continuous homomorphism dual to $f$. If $L$ is a topological group, $L_{0}$ will denote the identity component of $L$. A continuous homomorphism will be called proper if it is open onto its image. An exact sequence of continuous homomorphisms of

$$
A_{1} \stackrel{f_{1}}{\longrightarrow} A_{2} \stackrel{f_{2}}{\longrightarrow} \ldots \stackrel{f_{n-1}}{\longrightarrow} A_{n}
$$

is said to be proper exact if and only if $f_{i}$ is proper for each $i$. 
If $E: 0 \rightarrow L_{1} \stackrel{\alpha}{\rightarrow} L_{2} \stackrel{\beta}{\rightarrow} L_{3} \rightarrow 0$ is a proper short exact sequence of locally compact groups then by $\widehat{E}$ denote a proper short exact sequence $0 \rightarrow \widehat{L_{3}} \stackrel{\widehat{\beta}}{\rightarrow} \widehat{L_{2}} \stackrel{\widehat{\alpha}}{\rightarrow} \widehat{L_{1}} \rightarrow 0$. By $\boldsymbol{f}$ denote the forgetful functor from the category of locally compact groups to the category of discrete groups, which forgets the topological structure. By $\mathrm{Ext}_{Z}$ denote the extension functor on the category of discrete abelian groups. By Ext denote the extension functor on the category of locally compact abelian groups discussed by Fulp and Griffith [8]. Ext is an additive functor from $\mathcal{L} \times \mathcal{L}$ to $\mathcal{A}$. If $A$ and $B$ are locally compact groups, then $\operatorname{Ext}(A, B)$ is the (discrete) group of all extensions of $B$ by $A$. The functor Ext generalizes the functor Ext $_{Z}$. If $A$ and $B$ are discrete groups, then $\operatorname{Ext}(\mathrm{A}, \mathrm{B}) \cong \operatorname{Ext}_{Z}(A, B)$. If $A$ is discrete and $L$ is locally compact, then $\operatorname{Ext}(A, L) \cong \operatorname{Ext}_{Z}(A, \boldsymbol{f}(L))$. If $A$ and $B$ are locally compact groups, then there is the natural isomorphism $\operatorname{Ext}(A, B) \cong$ $\operatorname{Ext}(\widehat{B}, \widehat{A})$. Ext commutes with finite direct products in each arguments. By $\operatorname{Hom}(A, B)$ denote the (discrete) group of continuous homomorphisms from $A$ to $B$. There are long exact sequences which connect Hom to Ext.

By $\boldsymbol{S}$ denote the character group of the additive group of rational numbers. We denote by $\boldsymbol{R}$ the additive group of real numbers with its natural topology. Let $\boldsymbol{Z}_{p}$ be the additive group of $p$-adic integers with its natural topology. A discrete group is said to be algebraically compact if it is a direct summand of every group containing it as a pure subgroup. A group is algebraically compact if and only if it is an algebraically direct summand of some discrete group which admits a compact topology [4], Theorem $38.1 \mathrm{~d}$ ). Hence if $K$ is any compact group, then $\boldsymbol{f}(K)$ is algebraically compact. Every algebraically compact group is cotorsion.

If $L$ is any locally compact group then $L \cong \boldsymbol{R}^{n} \oplus M$, where $M$ contains an open compact subgroup ([6], Theorem 24.30).

\section{Dual cotorsion groups}

We shall that a compact group $K$ is called dual cotorsion if the character group $\widehat{K}$ of the group $K$ is a cotorsion group. We claim that a compact group $G$ is dual cotorsion if and only if $\operatorname{Ext}(G, S)=0$. Indeed, this follows from Pontrjagin duality and the definition of cotorsion group. From elementary properties of cotorsion groups we conclude that a compact group $G$ is a dual cotorsion group if and only if $G$ satisfies $\operatorname{Ext}(G, C)=0$ for all compact connected groups $C$. Thus if $K$ is any compact group, $C$ is its connected subgroup and $K / C$ is dual cotorsion, then $K \cong C \oplus(K / C)$. 
Denote by $\mathcal{C} \mathcal{T}$ and $\mathcal{D C}$ the classes of cotorsion groups and dual cotorsion groups respectively. From the definition of dual cotorsion group, properties of cotorsion groups (see the book [4], chapter 9, § 54) by Pontrjagin duality we have next statements.

1. $A \in \mathcal{C T} \Leftrightarrow \widehat{A} \in \mathcal{D C}$

2. The class of dual cotorsion groups is closed under taking subgroups.

Proof. Let $L$ be any subgroup of the dual cotorsion group $K$. The group $\widehat{L}$ is the epimorphic image of the group $\widehat{K}$, hence $\widehat{L}$ is cotorsion ([4], $\S 54, A)$. It follows that $L$ is dual cotorsion.

3. Let $L$ be any coreduced dual cotorsion group. The factor group $L / M$ is a dual cotorsion group if and only if the subgroup $M$ is coreduced.

Proof. The group $\widehat{L / M}$ is the subgroup of the group $\widehat{L}$ and the corresponding factor group is isomorphic to $\widehat{M}$. Hence $\widehat{L / M}$ is cotorsion if and only if $\widehat{M}$ is reduced ([4], $\S 54, \mathrm{~B})$. Therefore, $L / M$ is dual cotorsion if and only if $M$ is coreduced.

4. Let $L$ be any coreduced dual cotorsion group and let $\varphi$ be any continuous endomorphism of L. Then $\operatorname{ker} \varphi$ and $\operatorname{im} \varphi$ are dual cotorsion groups.

Proof. This statement follows from [4], § 54, C).

5. If $K$ is the subgroup of $L$ such that $K$ and $L / K$ are dual cotorsion, then $L$ is dual cotorsion.

Proof. The groups $\widehat{K}$ and $\widehat{L / K}$ are cotorsion, it follows that $\widehat{L}$ is cotorsion ([4], §54, D). Thus $L$ is dual cotorsion.

6. Let $\left\{G_{i}\right\}_{\{i \in I\}}$ be a family of discrete groups and let $\widehat{G}$ be the character group of the direct product $\prod_{i \in I} G_{i}$. Then the group $\widehat{G}$ is a dual cotorsion group if and only if each group $\widehat{G}_{i}$ is a dual cotorsion group.

Proof. This statement follows from [4], § 54, E).

7. Let $M$ be any dual cotorsion group and let $K$ be any compact group. Then the character group of $\operatorname{Hom}(M, K)$ is a dual cotorsion group. 
Proof. $\operatorname{Hom}(M, K) \cong \operatorname{Hom}(\widehat{K}, \widehat{M})$. The group $\operatorname{Hom}(\widehat{K}, \widehat{M})$ is cotorsion ([4], § 54, G), hence its character group is dual cotorsion.

8. Let $K$ be any dual cotorsion coreduced group. Then there is a natural isomorphism $\operatorname{Ext}\left(K, \prod_{p} \boldsymbol{Z}_{p}\right) \cong \widehat{K}$. Thus $K$ is isomorphic to the character group of the group $\operatorname{Ext}\left(K, \prod_{p} \boldsymbol{Z}_{p}\right)$.

Proof. Since $K$ is dual cotorsion coredused then $\widehat{K}$ is cotorsion reduced such that there is an isomorphism $\widehat{K} \cong \operatorname{Ext}(Q / Z, \widehat{K})([4], \S 54, \mathrm{I})$. Now, by Ext functor properties, we obtain $\operatorname{Ext}(Q / Z, \widehat{K}) \cong \operatorname{Ext}(K, \widehat{Q / Z}) \cong$ $\operatorname{Ext}\left(K, \prod_{p} \boldsymbol{Z}_{p}\right)$.

9. A compact group $K$ is a dual cotorsion group if and only if there is some compact group $M$ such that $K$ is a subgroup of $M$ and the character group of $M$ is an algebraically compact group.

Proof. Since $K$ is dual cotorsion then $\widehat{K}$ is cotorsion such that there exist an exact sequence $M_{1} \stackrel{\beta}{\rightarrow} \widehat{K} \rightarrow 0$ where the group $M_{1}$ is an algebraically compact [4], §54, the Proposition 54.1. We get the proper exact sequence $0 \rightarrow K \stackrel{\widehat{\beta}}{\rightarrow} \widehat{M}_{1}$. Denote by $M$ the group $\widehat{M}_{1}$. The character group of $M$ is an algebraically compact and $K$ is isomorphic to a subgroup of $M$.

10. Let $K$ be a compact connected group. Then $K$ is dual cotorsion if and only if $K$ is the character group of some algebraically compact group.

Proof. The group $\widehat{K}$ is torsion free. Hence $\widehat{K}$ is cotorsion if and only if $\widehat{K}$ is an algebraically compact group [4], the Corollary 54.5.

11. Let $K$ be a compact totally disconnected group. Then $K$ is dual cotorsion if and only if $K \cong \prod_{p}\left(\prod_{m_{p}} \boldsymbol{Z}_{p}\right) \bigoplus M$, where $M$ is a torsion compact
group.

Proof. The group $\widehat{K}$ is torsion, since $K$ is a totally disconnected group. It follows $\widehat{K}$ is cotorsion if and only if $\widehat{K} \cong \bigoplus_{p}\left(\bigoplus_{m_{p}} Z\left(p^{\infty}\right)\right) \bigoplus L$, where the group $L$ is bounded ([4], the Corollary 54.4). Thus $K \cong \prod_{p}\left(\prod_{m_{p}} \boldsymbol{Z}_{p}\right) \bigoplus \widehat{L}$. Denote $\widehat{L}$ by $M$. The group $M$ group is torsion compact group, such that it is dual to a bounded discrete group. 
The list of properties of dual cotorsion groups can be extended using the Pontrjagin duality. The classification of dual cotorsion groups will be discussed in section 5 .

\section{The properties of the class generalized cotorsion groups}

Definition 3.1 (Main definition). A locally compact group $L$ is called a generalized cotorsion group, if $L$ contains an open dual cotorsion subgroup $K$ such that the group $L / K$ is a cotorsion group; in other words there is a proper short exact sequence

$$
0 \rightarrow K \rightarrow L \rightarrow A \rightarrow 0
$$

where $K$ is a dual cotorsion group and $A$ is a cotorsion group.

Any cotorsion group and any dual cotorsion are examples of generalized cotorsion groups. The additive group of all $p$-adic number with its natural topology is a generalized cotorsion group.

Denote by $\mathcal{G C T}$ the class of generalized cotorsion groups.

LEMMA 3.2. A locally compact group $L$ is a generalized cotorsion group if and only if $\widehat{L}$ is a generalized cotorsion group; in other words the class of generalized cotorsion groups is closed under Pontrjagin duality.

Proof. Let $K$ be an open dual cotorsion subgroup of $L$ such that $L / K$ is a cotorsion group. Then there exists the proper short exact sequence

$$
E: 0 \rightarrow K \rightarrow L \rightarrow A \rightarrow 0
$$

Hence there exists the proper short exact sequence

$$
\widehat{E}: 0 \rightarrow \widehat{A} \rightarrow \widehat{L} \rightarrow \widehat{K} \rightarrow 0
$$

where $\widehat{A}$ is a dual cotorsion group and $\widehat{K}$ is a cotorsion group. Therefore $\widehat{L}$ is a generalized cotorsion group.

THEOREM 3.3. Let $L$ by a locally compact group and $L$ admits no subgroups isomorphic to $\boldsymbol{R}$. The following conditions are equivalent:

1) $L$ is a generalized cotorsion group;

2) $\operatorname{Ext}(Q, L)=\operatorname{Ext}(L, S)=0$;

3) $\operatorname{Ext}_{Z}(Q, \boldsymbol{f}(L))=\operatorname{Ext}_{Z}(Q, \boldsymbol{f}(\widehat{L}))=0$; 
4) $\boldsymbol{f}(L)$ and $\boldsymbol{f}(\widehat{L})$ are cotorsion groups;

5) $\operatorname{Ext}(A, L)=\operatorname{Ext}(L, C)=0$ for all discrete torsion free groups $A$ and for all compact connected groups $C$.

Proof. 1) $\Rightarrow 2$ ). Let $K$ be an open dual cotorsion subgroup of $L$, such that $L / K$ is a cotorsion group. Consider the proper short exact sequence

$$
0 \rightarrow K \rightarrow L \rightarrow L / K \rightarrow 0
$$

We obtain the exact sequence

$$
\operatorname{Ext}(Q, K) \rightarrow \operatorname{Ext}(Q, L) \rightarrow \operatorname{Ext}(Q, L / K) \rightarrow 0
$$

Since $L / K$ is a cotorsion group we obtain

$$
\operatorname{Ext}(Q, L / K) \cong \operatorname{Ext}_{Z}(Q, L / K)=0
$$

The group $\boldsymbol{f}(K)$ is algebraically compact, therefore $\boldsymbol{f}(K)$ is a cotorsion group and we have

$$
\operatorname{Ext}(Q, K) \cong \operatorname{Ext}_{Z}(Q, \boldsymbol{f}(K))=0
$$

From (3.2) we get $\operatorname{Ext}(Q, L)=0$.

From Lemma 3.2 it follows that $\widehat{L}$ is a generalized cotorsion group and we obtain $\operatorname{Ext}(Q, \widehat{L})=0$. Since $\operatorname{Ext}(Q, \widehat{L})=0$ and $\operatorname{Ext}(L, S) \cong \operatorname{Ext}(Q, \widehat{L})$, we see that $\operatorname{Ext}(L, S)=0$.

$2) \Rightarrow 1$ ). Let $K$ be any compact open subgroup of the group $L$. Then we have proper exact sequences (3.1) and (3.2). By $\operatorname{Ext}(Q, L)=0$, so that $\operatorname{Ext}(Q, L / K)=0$. It follows that the discrete group $L / K$ is cotorsion. The proper short exact sequence (3.1) yields the exact sequence

$$
\operatorname{Ext}(L / K, \boldsymbol{S}) \rightarrow \operatorname{Ext}(L, \boldsymbol{S}) \rightarrow \operatorname{Ext}(K, \boldsymbol{S}) \rightarrow 0
$$

$\operatorname{By} \operatorname{Ext}(L, \boldsymbol{S})=0$, so that $\operatorname{Ext}(K, \boldsymbol{S})=0$, i.e. the group $K$ is dual cotorsion. From Definition 3.1 it follows that $L$ is a generalized cotorsion group.

$2) \Leftrightarrow 3)$. Since $Q$ is a discrete group we get $\operatorname{Ext}(Q, L) \cong \operatorname{Ext}_{Z}(Q, \boldsymbol{f}(L))$. Also, $\operatorname{Ext}(L, S) \cong \operatorname{Ext}(\widehat{\boldsymbol{S}}, \widehat{L}) \cong \operatorname{Ext}(Q, \widehat{L}) ; \operatorname{thus} \operatorname{Ext}(L, \boldsymbol{S}) \cong \operatorname{Ext}_{Z}(Q, \boldsymbol{f}(\widehat{L}))$.

$3) \Leftrightarrow 4)$. By definition of cotorsion group we conclude that this equivalence is true.

$4) \Leftrightarrow 5$ ). From elementary properties of cotorsion groups and Pontrjagin duality it follows that this equivalence is true.

Corollary 3.4 and Corollary 3.5 follows immediately from Theorem 3.3. 
COROLLARY 3.4. If $L$ is any generalized cotorsion group and $L$ is an open subgroup of a locally compact group $M$ such that $M / L$ is torsion free, then $L$ is a direct summand of $M$.

Corollary 3.5. If $L$ is any locally compact group and $C$ is compact connected subgroup of $L$ such that $L / C$ is a generalized cotorsion group, then $C$ is a direct summand of the group $L$.

Proposition 3.6. 1) Let $A$ be any discrete group. Then $A$ is generalized cotorsion if and only if $A$ is cotorsion, i.e. $\mathcal{C T}=\mathcal{A} \cap \mathcal{G C T}$. Thus a discrete group $A$ is generalized cotorsion if and only if $\operatorname{Ext}(Q, A)=0$.

2) Let $K$ be any compact group. Then $K$ is generalized cotorsion if and only if $K$ is dual cotorsion, i.e. $\mathcal{D C}=\mathcal{C} \cap \mathcal{G C T}$. Thus a compact group $K$ is generalized cotorsion if and only if $\operatorname{Ext}(K, \boldsymbol{S})=0$.

Proof. 1) Let $A$ be any discrete group such that $A$ is generalized cotorsion. From Theorem 3.34 ) it follows that $A$ is cotorsion. Conversely, if $A$ is any cotorsion group, then $\operatorname{Ext}(Q, A)=0$. Since $\boldsymbol{f}(\widehat{A})$ is an algebraically compact group, it follows that $\boldsymbol{f}(\widehat{A})$ is a cotorsion group. Since $\boldsymbol{f}(\widehat{A})$ is a cotorsion group, we have $\operatorname{Ext}(A, \boldsymbol{S})=\operatorname{Ext}(Q, \widehat{A})=\operatorname{Ext}_{Z}(Q, \boldsymbol{f}(\widehat{A}))=0$ and $A$ is a generalized cotorsion group.

2) can be obtained from 1) by Pontrjagin duality.

THEOREM 3.7. The class of generalized cotorsion locally compact groups is closed under taking:

1) extensions;

2) finite direct sums;

3) topologically direct summands;

4) compact subgroups;

5) discrete epimorphic images.

Proof. 1) Let $0 \rightarrow K \rightarrow L \rightarrow M \rightarrow 0$ be a proper short exact sequence such that $K$ and $M$ are generalized cotorsion groups. We will prove that $L$ is a generalized cotorsion group too. We have the exact sequences

$$
\operatorname{Ext}(Q, K) \rightarrow \operatorname{Ext}(Q, L) \rightarrow \operatorname{Ext}(Q, M) \rightarrow 0
$$

and

$$
\operatorname{Ext}(M, \boldsymbol{S}) \rightarrow \operatorname{Ext}(L, S) \rightarrow \operatorname{Ext}(K, \boldsymbol{S}) \rightarrow 0
$$

Since $\operatorname{Ext}(Q, K)=\operatorname{Ext}(Q, M)=\operatorname{Ext}(M, S)=\operatorname{Ext}(K, \boldsymbol{S})=0$, we get $\operatorname{Ext}(Q, L)=\operatorname{Ext}(L, S)=0$. Thus $L$ is generalized cotorsion. 
The properties 2) and 3) follow from Theorem 3.3 since Ext commutes with finite direct products.

4) Let $L$ be any generalized cotorsion group and let $K$ be an arbitrary compact subgroup of $L$. The proper short exact sequence

$$
0 \rightarrow K \rightarrow L \rightarrow L / K \rightarrow 0
$$

yields the exact sequence

$$
\operatorname{Ext}(L / K, \boldsymbol{S}) \rightarrow \operatorname{Ext}(L, \boldsymbol{S}) \rightarrow \operatorname{Ext}(K, \boldsymbol{S}) \rightarrow 0
$$

Using $\operatorname{Ext}(L, \boldsymbol{S})=0$, we get $\operatorname{Ext}(K, \boldsymbol{S})=0$. From Proposition 3.6 it follows that $K$ is generalized cotorsion.

5) Let $L$ be any generalized cotorsion group and let $M$ be a discrete epimorphic image of $L$. From the proper exact sequence $L \rightarrow M \rightarrow 0$ we have the exact sequence $\operatorname{Ext}(Q, L) \rightarrow \operatorname{Ext}(Q, M) \rightarrow 0 . \operatorname{By} \operatorname{Ext}(Q, L)=0$, so that $\operatorname{Ext}(Q, M)=0$. From Proposition 3.6 it follows that $M$ is a generalized cotorsion group.

\section{Some homological properties of generalized cotorsion groups}

Let $A$ be any discrete group. Then $A$ is cotorsion if and only if $A$ has the injective property relative to all torsion splitting short exact sequences ([4], chapter 9, the Theorem 58.2). Remind that a short exact sequence $E: 0 \rightarrow A \rightarrow B \rightarrow C \rightarrow 0$ of discrete groups is called torsion splitting if the sequence $E \tau$ is splitting, where $\tau: T(C) \rightarrow C$ is the natural inclusion. The notion of compact splitting sequence, that we introduce in next definition, is a suitable generalization of the concept of torsion splitting sequence.

Let $M$ be any locally compact group. Denote by $B(M)$ the subgroup of $M$ such that $c \in B(M)$ if and only if $c$ is a compact element. An element $c$ is called compact if the smallest closed subgroup which contains the element $c$ is compact ([6], chapter 2, the Definition 9.9).

DeFinition 4.1. A proper short exact sequence of locally compact groups

$$
E: 0 \rightarrow K \rightarrow L \rightarrow M \rightarrow 0
$$

is called compact splitting if the sequence $E \tau$ is splitting, where $\tau$ is the natural inclusion $\tau: B(M) \rightarrow M$.

Let $E: 0 \rightarrow K \rightarrow L \rightarrow M \rightarrow 0$ be any proper short exact sequence of locally compact groups. Obviously, if the group $M$ has no compact elements then $E$ is compact splitting. 
Let $A$ be any discrete group. Then an element $a \in A$ is compact if and only if $a$ has a finite order. Therefore, if $A$ is a discrete group then $T(A)=B(A)$ and we conclude that a short exact sequence $E$ of discrete groups is compact splitting if and only if $E$ is torsion splitting.

Now we introduce a new concept. It is dual to the notion of compact splitting sequence.

DEFINITION 4.2. A proper short exact sequence of locally compact groups

$$
E: 0 \rightarrow K \rightarrow L \rightarrow M \rightarrow 0
$$

is called $t d$-splitting (totally disconnected splitting) if the sequence $\sigma E$ is splitting, where $\sigma$ is the natural projection $\sigma: K \rightarrow K / K_{0}$.

Let $E: 0 \rightarrow K \rightarrow L \rightarrow M \rightarrow 0$ be any proper short exact sequence of locally compact groups. Evidently, if the group $K$ is connected then $E$ is $t d$-splitting. If the group $K$ is totally disconnected then $E$ is $t d$-splitting if and only if $E$ is splitting. Hence if $E$ is a short exact sequence of discrete groups, then $E$ is $t d$-splitting if and only if $E$ is splitting.

Let $M$ be any locally compact group and let $B(M)$ be the subgroup of compact elements of $M$. Evidently, if $E: 0 \rightarrow B(M) \stackrel{\tau}{\rightarrow} M$ is a proper exact sequence, then $\widehat{E}: \widehat{M} \stackrel{\widehat{\tau}}{\rightarrow} \widehat{M} / \widehat{M}_{0} \rightarrow 0$ is a proper exact sequence too, by Pontrjagin duality.

From the last remarks by Pontrjagin duality we obtain the next lemma.

Lemma 4.3. 1) A proper short exact sequence $E$ of locally compact groups is compact splitting if and only if the sequence $\widehat{E}$ is td-splitting.

2) Let $L$ be any locally compact group. Then $L$ has the injective (projective) property relative to all compact splitting sequences if and only if $\widehat{L}$ has the projective (injective) property relative to all $t d$-splitting sequences.

THEOREM 4.4. Let $L$ be any generalized cotorsion locally compact group. Then $L$ has the injective property relative to all compact splitting sequences and $L$ has the projective property relative to all td-splitting sequences.

Proof. Let $E: 0 \rightarrow L_{1} \stackrel{\alpha}{\rightarrow} L_{2} \stackrel{\beta}{\rightarrow} L_{3} \rightarrow 0$ be any proper short exact compact splitting sequence of locally compact groups and $\varphi: L_{1} \rightarrow L$ be any continuous homomorphism. Suppose first, that $B\left(L_{3}\right)=0$. Then $L_{3}$ has no 
compact subgroups and we get $L_{3} \cong \boldsymbol{R}^{n} \bigoplus A$, where $A$ is a discrete torsion free group. We get $\operatorname{Ext}\left(L_{3}, L\right) \cong \operatorname{Ext}\left(\boldsymbol{R}^{n}, L\right) \bigoplus \operatorname{Ext}(A, L)=0$. The short exact sequence $E$ yields the exact sequence

$$
\operatorname{Hom}\left(L_{2}, L\right) \stackrel{\alpha^{*}}{\rightarrow} \operatorname{Hom}\left(L_{1}, L\right) \rightarrow \operatorname{Ext}\left(L_{3}, L\right)=0
$$

It follows that $\alpha^{*}$ is an epimorphism. Hence every continuous homomorphism from $L_{1}$ to $L$ can be extended to a continuous homomorphism from $L_{2}$ to $L$.

Let $B\left(L_{3}\right) \neq 0$ and let $\tau$ be a natural inclusion $\tau: B\left(L_{3}\right) \rightarrow L_{3}$, then $E \tau$ is splitting.

We get the following commutative diagram:

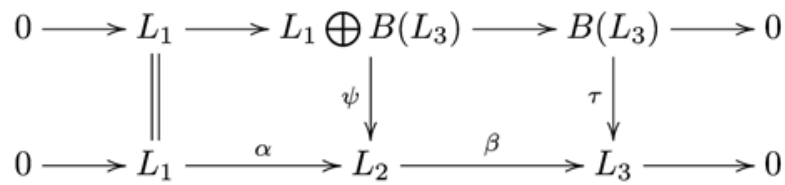

Define the map $\varphi_{1}: L_{1} \bigoplus B\left(L_{3}\right) \rightarrow L$ by the rule $\varphi_{1}((a, b))=\varphi(a)$ for every $a \in L_{1}$. Clearly the map $\varphi_{1}$ is a continuous homomorphism and the homomorphism $\varphi_{1}$ extends the homomorphism $\varphi$. Let $L_{2}^{\prime}$ be the inverse image of subgroup $B\left(L_{3}\right)$ relative $\beta$. By construction of induced extension we conclude each $x \in L_{2}^{\prime}$ has a unique inverse image relatively $\psi$. It is to prove that there exists the continuous homomorphism $\varphi_{2}: L_{2}^{\prime} \rightarrow L$, such that we get $\varphi=\varphi_{2} \alpha$.

Denote by $L_{3}^{\prime}$ the factor group $L_{2} / L_{2}^{\prime}$. The group $L_{3}^{\prime}$ has no compact elements and we have the proper short exact sequence

$$
0 \rightarrow L_{2}^{\prime} \rightarrow L_{2} \rightarrow L_{3}^{\prime} \rightarrow 0
$$

It follows that there exists a continuous homomorphism $\varphi_{3}: L_{2} \rightarrow L$ such that $\varphi_{3}$ extends $\varphi_{2}$, therefore we see that $\varphi=\varphi_{3} \alpha$. It follows that the group $L$ has the injective property to all compact splitting sequences.

Since the group $\widehat{L}$ is generalized cotorsion too, then $\widehat{L}$ has an injective property to all compact splitting sequences. From Lemma 4.3 it follows that the group $L$ has the projective property to all $t d$-split short exact sequences.

Every reduced discrete group $A$ can be embedded in a reduced cotorsion group $C(A)$ such that $C(A) / A$ is torsion free and divisible. Moreover, this embedding is unique in the sense that if $A$ is contained in a reduced cotorsion group $B$ with $B / A$ torsion free and divisible then there is an 
isomorphism $\psi$ between $C(A)$ and $B$ which preserves the identity map on $A$. The group $C(A)$ is called the cotorsion envelope of $A$ (see [4], chapter 9 , the Theorem 58.1). There is an isomorphism $C(A) \cong \operatorname{Ext}_{Z}(Q / Z, A)$.

Now we introduce a new concept. A coreduced compact group $D C(K)$ is called a dual cotorsion covering of the compact coreduced group $K$ if the following conditions hold:

i) $D C(K)$ is dual cotorsion and coreduced;

ii) there is an open continuous epimorphism $\varphi: D C(K) \rightarrow K$;

iii) $\operatorname{ker} \varphi \cong \boldsymbol{S}^{\alpha}$.

By Pontrjagin duality we conclude that $D C(K)$ is isomorphic to the character group of group $\operatorname{Ext}\left(K, \prod_{p} Z_{p}\right)$.

THEOREM 4.5. Let $K$ by any compact coreduced group and $A$ by any discrete reduced group. If $C(A)$ is the cotorsion envelope of $A$ and $D C(K)$ is the dual cotorsion covering of $K$, then there is a natural isomorphism $\operatorname{Ext}(A, K) \cong \operatorname{Ext}(C(A), D C(K))$.

Proof. From the short exact sequence of discrete groups

$$
0 \rightarrow A \rightarrow C(A) \rightarrow C(A) / A \rightarrow 0
$$

we have the exact sequence

$$
\operatorname{Ext}(C(A) / A, K) \rightarrow \operatorname{Ext}(C(A), K) \rightarrow \operatorname{Ext}(A, K) \rightarrow 0,
$$

Since $K$ is a compact group and $C(A) / A$ is a torsion free discrete group, then we get $\operatorname{Ext}(C(A) / A, K)=0$ ([9], Corollary 2.3) and we have an isomorphism

$$
\operatorname{Ext}(C(A), K) \cong \operatorname{Ext}(A, K)
$$

From the proper short exact sequence of compact groups

$$
0 \rightarrow \boldsymbol{S}^{\alpha} \rightarrow D C(K) \rightarrow K \rightarrow 0
$$

we have the exact sequence

$$
\operatorname{Ext}\left(C(A), S^{\alpha}\right) \rightarrow \operatorname{Ext}(C(A), D C(K)) \rightarrow \operatorname{Ext}(C(A), K) \rightarrow 0,
$$

Since $\boldsymbol{S}^{\alpha}$ is a compact connected group and $C(A)$ is a discrete group, we get $\operatorname{Ext}\left(C(A), \boldsymbol{S}^{\alpha}\right)=0$ ([9], Corollary 2.4). From the last exact sequence we have

$$
\operatorname{Ext}(C(A), D C(K)) \cong \operatorname{Ext}(C(A), K)
$$

Using (4.1) and (4.2), we get $\operatorname{Ext}(C(A), D C(K)) \cong \operatorname{Ext}(A, K)$. This completes the proof of Theorem 4.5. 
Theorem 4.5 shows that generalized cotorsion groups play an exceptional role in the structural theory of locally compact groups. Really, if $K$ is any compact coreduced group and $A$ is any reduced discrete group, then there is one-to-one correspondence between the classes of extensions $K$ by $A$ and the classes of extensions $D C(K)$ by $C(A)$. Every extension $D C(K)$ by $C(A)$ is a generalized cotorsion group.

\section{Some results about a classification of generalized cotorsion groups}

We recall now what is observed in [4] that if $A$ is any cotorsion group then $A \cong D \oplus C \oplus F$, where $D$ is a divisible group, $C$ is a reduced torsion free algebraically compact group and $F$ is an adjusted group. A reduced cotorsion group $F$ is called adjusted if $F$ admits no nonzero torsion free direct summand. Every divisible group and every algebraically compact group are characterized by cardinal invariants (see [4], chapter 4 and chapter 7). The correspondence $\varphi: T \mapsto \operatorname{Ext}_{Z}(Q / Z, T)$ is a bijective map from the class of reduced torsion groups in the class of adjusted cotorsion groups (Harrison, [1]). The inverse mapping $\varphi^{-1}$ is taking the torsion part of the adjusted cotorsion group. Therefore any adjusted cotorsion group $F$ is characterized by its torsion part. The problem of the classification of torsion groups is reduced to the problem of classification of primary torsion groups. For example the groups from the class of totally projective groups are characterized by cardinal invariants. These are well-known Ulm invariants (see, [5], chapter 12, §82). Another class of p-primary groups characterized by cardinal invariants is the class of torsion complete groups (see, [5], chapter 11, § 68).

We say that a coreduced dual cotorsion group $C$ is called coadjusted if its character group $\widehat{C}$ is an adjusted group. From Pontrjagin duality we conclude that the correspondence $\psi: C \rightarrow C / C_{0}$ is a bijective mapping from the class of coadjusted groups in the class of coreduced totally disconnected groups. It follows that if $C$ is any coadjusted group, then $C$ has the same invariants as the quotient group $C / C_{0}$. Let $K$ be any dual cotorsion group. Then there is an isomorphism $K \cong E \bigoplus A_{k} \bigoplus C$, where $E$ is a torsion free compact group (i.e. $E$ is a character group of a divisible group) and $A_{k}$ is a character group of a reduced torsion free algebraically compact group, and $C$ is coadjusted. The groups $E$ and $A_{k}$ may be characterized by cardinal invariants. A coadjusted group $C$ can be characterized by cardinal invariants in some cases. For example, suppose that every topologically $p$-primary component of the group $C / C_{0}$ is dual to some to- 
tally projective group. Then the topologically $p$-primary components of $C / C_{0}$ has the cardinal invariants. There are co-Ulm invariants, where Kiefer [10] introduced, (see also [7], chapter 5). A lot of these cardinal invariants for all prime $p$ will be the system invariants of the group $C$.

Let $K$ be any compact group and let $A$ be any discrete group, and let $P$ be the set of all prime numbers. Denote by $T_{p}(A)$ the $p$-primary component of the torsion part of the group $A$ and $\pi(A)=\left\{p \in P \mid T_{p}(A) \neq 0\right\}$. Let $q(K)$ be the set $\{q \in P \mid q K \neq K\}$. In [9], is proved that $\operatorname{Ext}(A, K)=0$ if and only if $\pi(A) \cap q(K)=\emptyset$. The last equality is equivalent to $\pi(A) \cap \pi(\widehat{K})=\emptyset$. Therefore if $L$ is any generalized cotorsion group and $L$ is the extension of the compact group $K$ by the discrete group $A$, and $\pi(A) \cap \pi(\widehat{K})=\emptyset$ then $L \cong K \bigoplus A$. This yields that the invariants of the groups $K$ and $A$ are the invariants of the group $L$ in this case.

\section{REFERENCES}

[1] D. HARRISON, Infinite abelian groups and homological methods, Ann. Math., 69 (1959), pp. 366-409.

[2] L. Fuchs, Notes on abelian groups, I, Ann. Unniv. Sci. Budapest., 2 (1959), pp. 5-23; II, Acta. Math. Acad. Sci. Hungar., 11 (1960), pp. 117-125.

[3] R. J. Nunke, Modules of extensions over Dedekind rings, Ill. J. Math., 3 (1959), pp. 222-241.

[4] L. Fuchs, Infinite abelian groups. V. 1, Academic Press, 1970.

[5] L. Fuchs, Infinite abelian groups. V. 2, Academic Press, 1973.

[6] E. HewitT - K. Ross, Abstract Harmonic Analysis. V. 1, Springer, 1975.

[7] P. Lотн, Classification of Abelian groups and Pontrjagin Duality. Gordon and Breach Science Publishers, 1998.

[8] R. FulP - Ph. GRIFFITH, Extensions of locally compact abelian groups I, II, Trans. Amer. Math. Soc., 154 (1971), pp. 341-363.

[9] N. I. KRYuchKov, On the abelian extensions of locally compact abelian groups, Math. USSR Sb, 41 (1982), pp. 511-522.

[10] F. KIEFER, The duals of totally projective groups, Lecture Notes in Math., 874 (1981), pp. 297-304.

Manoscritto pervenuto in redazione il 27 marzo 2011. 